\title{
Structure-Dependent Hydrostatic Deformation Potentials of Individual Single- Walled Carbon Nanotubes
}

\author{
J. Wu ${ }^{1 *}$, W. Walukiewicz ${ }^{1}$, W. Shan ${ }^{1}$, E. D. Bourret-Courchesne ${ }^{1}$, J. W. Ager $\mathrm{III}^{1}$, K. M. \\ $\mathrm{Yu}^{1}$, E. E. Haller ${ }^{1,2}$, Kyle Kissell $^{3}$, Sergei M. Bachilo ${ }^{3}$, R. Bruce Weisman ${ }^{3}$ and Richard E. \\ Smalley $^{3}$
}

1. Materials Sciences Division, Lawrence Berkeley National Laboratory, Berkeley, California 94720

2. Department of Materials Science and Engineering, University of California, Berkeley, California 94720

3. Department of Chemistry, Rice University, 6100 Main St., Houston, Texas 77005

The hydrostatic pressure coefficients of interband transition energies of a number of single-walled carbon nanotubes with different chiralities were measured. Both optical absorption and photoluminescence experiments were performed on de-bundled, singlewalled carbon nanotube suspensions with hydrostatic pressure applied by diamond anvil cells. The pressure coefficients of the first van Hove transition (bandgap) energies are negative and dependent on the nanotube structure, while the second van Hove transitions are much less sensitive to hydrostatic pressure. The hydrostatic deformation potentials of individual nanotubes are deduced within an elastic model. An empirical equation that relates the pressure coefficients to nanotube structure is presented and discussed.

PACS numbers: 78.67.Ch, 62.25.+g, 71.20.Tx, 81.07.De

*Electronic Mail: jqwu@ux8.lbl.gov 
The unique electronic and mechanical properties and the prospects for technological applications of single-walled carbon nanotubes (SWNTs) have led to considerable research interest. Nanotubes can be either metallic or semiconducting depending on their chiral structure, which is indexed by two integers $(n, m)$ [1]. SWNTs have been also shown to have superior elastic properties that can accommodate large mechanical strains [2]. A more detailed understanding of the electromechanical responses of SWNTs is important in view of the practical manipulation of SWNTs. It has been predicted theoretically that under uniaxial stretch the bandgap of a semiconducting SWNT should increase or decrease depending on whether $(n-m)$ mod 3 (the remainder of dividing $n-m$ by 3 ) is equal to 1 or 2 [3]. Experimental results have been shown to be consistent with these predictions [4-6]. For example, very recently Minot et. al. found that the conductance of SWNTs is modulated by the axial tensile strain introduced by pushing the middle of a suspended nanotube with an Atomic Force Microscope tip [5]. The results were explained by a $53 \mathrm{meV} / \%$-stretch decrease in the bandgap of a semiconducting nanotube and a $35 \mathrm{meV} / \%$-stretch increase in the bandgap of a metallic nanotube. Similar results have also been reported on metallic and quasi-metallic nanotubes [6]. However, all of these studies have been based on uniaxial tensile deformation along the tube axis. They have neglected the local distortion of the nanotubes by the touch of the AFM tip or the electrodes and have assumed a simultaneous contraction in the tube radial direction in accord with the Poisson's ratio [5$6]$.

Experimental investigations of SWNTs under hydrostatic pressure have been limited to bundled nanotube ropes $[7,8]$. In these studies, the detected signals include not only contributions from the intrinsic response of individual nanotubes but also contributions from the inter-tube interactions within a bundle that are enhanced under pressure $[7,8]$. These inter-tube interactions can cause considerable extrinsic, anisotropic effects on the individual nanotubes such as a polygonization of the tube cross section [8].

In general, bundled SWNTs do not fluoresce. Very recently, bright band-edge fluorescence has been observed from semiconducting SWNTs suspended by aqueous sodium dodecyl sulfate (SDS) surfactant in deuterium oxide $\left(\mathrm{D}_{2} \mathrm{O}\right)$ and treated by intensive ultrasonication and subsequent centrifugation [9]. The resultant suspension is 
rich in dispersed individual SWNTs that are in van der Waals contact with the surrounding SDS surfactant coating, which is presumed to reduce the fluorescence quenching effects by isolating semiconducting nanotubes from metallic ones. This technique provides a unique way to study the intrinsic hydrostatic pressure behavior of individual nanotubes by optical spectroscopies. In this letter we present our systematic measurements of the hydrostatic pressure dependence of optical transitions in individual SWNTs. The deformation potentials of nanotube bandgaps are derived within an elastic model. The pressure coefficients for different nanotube structures can be expressed as the summation of a diameter-dependent term and a chiral angle-dependent term.

A nanotube suspension containing $25.2 \mathrm{mg} / 1$ of SWNTs grown in high-pressure carbon monoxide was processed in a similar way as described in Ref. [9]. The optical absorption measurements were performed at room temperature using a halogen tungsten lamp dispersed by a $0.5 \mathrm{~m}$ monochromator as the light source. A Ge photodiode was used as the detector in the spectral range below $1.4 \mathrm{eV}$, and an ultraviolet-enhanced $\mathrm{Si}$ photodiode was used for energies above $1.4 \mathrm{eV}$. For photoluminescence (PL) measurements, the PL signals were generated in the backscattering geometry by excitation with the $476.5 \mathrm{~nm}$ line of an argon laser. The emission was dispersed in a monochromator and directed to a liquid nitrogen-cooled Ge detector. For pressuredependent optical experiments, a drop of nanotube suspension was sealed in a gasketed diamond anvil cell for the application of hydrostatic pressure. The pressurized volume was a cylindrical-shaped hole (diameter $\sim 150 \mu \mathrm{m}$ and length $\sim 200 \mu \mathrm{m}$ ) drilled into the spring steel gasket sheet. A small grain of ruby $(\sim 10 \mu \mathrm{m}$ grain size $)$ was also loaded in the volume. The applied pressure was calibrated by the standard method of monitoring the red shift of the ruby R1 emission line. A spectrum taken from a nanotube-free SDS$\mathrm{D}_{2} \mathrm{O}$ suspension was used as the reference signal.

Figure 1 shows the ambient-pressure absorption spectrum of the sample obtained on a CARY-2390 NIR-VIS-UV spectrophotometer after the featureless background baseline had been subtracted. A series of absorption peaks, similar to those reported in Ref. [9], are resolved distinctly. The peaks can be divided into two groups. In the energy region below $\sim 1.4 \mathrm{eV}$, twelve absorption peaks (group A transitions) are observed with full width at half maximum (FWHM) between $10 \mathrm{meV}$ and $30 \mathrm{meV}$. In the energy region 
between $1.4 \mathrm{eV}$ and $3.1 \mathrm{eV}$, there is another set of twelve peaks (group B transitions) with FWHM between $50 \mathrm{meV}$ and $100 \mathrm{meV}$. For convenience of discussion, we number these peaks in the order of increasing peak energy (0.899 to $3.030 \mathrm{eV})$, as shown in Fig. 1. In addition to the difference of peak width, these two groups of transitions also exhibit completely different emission behavior. As is seen in the inset of Fig. 1, photo-excitation of the sample results in strong emission in the spectral range of the group A transitions, with each PL peak position well matched to an individual absorption peak [10]. In contrast, in the spectral range of group B transitions, no characteristic emission was detected (data not shown here) [11].

The different characteristics between group A and group B are attributed to the different nature of optical transitions involved [12]. Group A peaks correspond to optical transitions across the bandgap of the semiconducting nanotubes between the lowest unoccupied van Hove singularity (VHS) and the highest occupied VHS, and are conventionally denoted as $\mathrm{E}_{11}$. Group $\mathrm{B}$ peaks $\left(\mathrm{E}_{22}\right)$ are attributed to the transitions between the second pair of VHS above and below the Fermi level, respectively. Upon photo-excitation with energies above $\mathrm{E}_{22}$, the photo-generated charge carriers thermalize to the first pair of VHS. Subsequent radiative carrier recombination results in band-edge emission at the energy of $E_{11}$. Similar clustering and attribution of absorption peaks have also been discussed in Ref. [13].

Using an excitation-emission spectrofluoimetric mapping, Bachilo et. al. have assigned each optical transition to a specific SWNT structure [12]. The equation that best relates the nanotube geometry and the optical transition energies is given by [12],

$$
E_{i i}=\frac{A_{i i}}{B_{i i}+d}+\frac{C_{i i} \cdot \cos 3 \alpha}{d^{2}},
$$

where $i=1$ or $2 . d$ and $\alpha$ are the nanotube diameter and the chiral angle, respectively. $A_{i i}$, $B_{i i}$ and $C_{i i}$ are empirical constants given in Ref. [12]. The sign and the numerical value of $C_{i i}$ also depend on the value of $q=(n-m) \bmod 3$. Using Eq.(1) we have assigned a pair of $(n, m)$ structure indices to each peak in group A and group B. The assignment is listed in Table I. The fact that several $E_{11}$ peaks correspond to the same $E_{22}$ peak indicates that the broad $\mathrm{E}_{22}$ peak includes contributions from several different nanotube structures. Peaks 
18, 21, 22 and 23 do not match any $E_{11}$ peaks observed in the range. They are attributed to the lowest-energy van Hove transitions of metallic nanotubes in the sample [12].

The effect of hydrostatic pressure on the absorption spectrum of group A peaks is shown in Fig. 2(a). All peaks shift to lower energy with increasing hydrostatic pressure, opposite to most conventional direct-gap semiconductors. The width, line-shape and intensity of all peaks are well maintained from that at ambient pressure up to $8.5 \mathrm{kbar}$, at which the peaks start to weaken and broaden. At pressures above $\sim 11 \mathrm{kbar}$, the sample turns from a homogenous liquid into a multi-grain-structured phase and the resultant light scattering severely reduces the optical signal. This phase transition is related to the wellknown crystallization of $\mathrm{D}_{2} \mathrm{O}$ into a tetragonal solid phase (water VI) at $11 \mathrm{kbar}$ at room temperature [14]. The insensitivity of the line-shape of absorption peaks to the hydrostatic pressure leads us to believe that the extended electronic states in the SWNTs are not substantially perturbed by the van der Waals interactions between the surfactant coating and the nanotubes. Similar to the pressure dependence of absorption, the $\mathrm{E}_{11} \mathrm{PL}$ peaks also shift to lower energy under pressure (not show here). The PL peak and the corresponding absorption peak show very similar pressure dependence. A continuous reduction in PL intensity was also observed with increasing pressure. The PL intensity reduction is possibly caused by non-radiative recombination centers induced on the surface of the nanotubes by the interaction with the surfactant coating under pressure. As in conventional semiconductors the introduction of non-radiative defects effectively reduces the emission efficiency, while leaving the absorption between extended states largely unaffected.

In Fig. 2(b) we show the absorption spectrum of the group B transitions under various hydrostatic pressures. Unlike the group A absorption peaks, most of the group B peaks shift only slightly to lower energy. The line-shapes of all peaks also remain unaffected within the hydrostatic pressure range. The pressure coefficient of each peak is obtained from a least-square linear fitting to the pressure dependence of the absorption peak, which is shown in Fig. 3 and listed in Table I.

A few conclusions can be drawn from the results in Fig. 3. First of all, the application of hydrostatic pressure to SWNTs always decreases the bandgap $\left(\mathrm{E}_{11}\right)$ irrespective of whether the value of $q$ is equal to 1 or 2 . This behavior is in contrast to the 
effects of uniaxial stress where both decrease and increase of the bandgap are possible depending on the value of $q[5,6]$. The pressure coefficients for $\mathrm{E}_{22}$ transitions are considerably smaller than those of $E_{11}$ transitions. We note that a similar, overall shift to lower energy ( $\sim-2 \mathrm{meV} / \mathrm{kbar}$ below $10 \mathrm{kbar})$ of a broad absorption peak under hydrostatic pressure has been reported on bundled nanotubes [7]. Our studies show that the negative pressure coefficient is an intrinsic property of individual nanotubes, rather than an effect of inter-tube interactions within a bundle under high pressures.

Due to the highly anisotropic geometry of nanotubes, their mechanical properties are different in the axial direction and the radial direction. By performing first-principles calculations of the elastic constants of individual nanotubes, Reich et. al. have found that the linear modulus in the axial direction $(|d P / d \ln z|)$ is 1.65 times larger than in the radial direction $(|d P / d \ln d|)$ [15]. The linear moduli are insensitive to chirality for nanotubes with the same diameter. A continuum model within classical elasticity theory was also introduced to model the linear and bulk moduli of SWNTs, which shows good agreement with the first-principles calculations [15]. The bulk modulus of individual nanotubes with diameter $d$ is given in the elastic model by [15]

$$
\frac{d P}{d \ln V}=-\frac{4 Y}{1-2 v} \cdot \frac{w \cdot d}{(d+w)^{2}}\left[3+2 \frac{1+v}{1-2 v}\left(\frac{d-w}{d}\right)^{2}\right]^{-1},
$$

in which $Y \approx 1 \mathrm{TPa}$ is the Young's modulus, $v \approx 0.14$ is the Poisson's ratio, and $w=0.31$ $\mathrm{nm}$ is the effective nanotube wall thickness. The hydrostatic deformation potential of the nanotube bandgap can be calculated from $d E_{11} / d \ln V=\left(d E_{11} / d P\right) \cdot(d P / d \ln V)$. The calculated results are listed in Tab. I. The magnitude of deformation potentials is much smaller than that of diamond $(25 \mathrm{eV})$, but comparable to that of most other compound semiconductors [16].

Another striking effect shown in Fig. 3 is that the magnitude of pressure coefficients of the group A peaks ( $\left.\left|d E_{11} / d P\right|\right)$ tends to increase with decreasing transition energy. Since the main term in $E_{11}$ is inversely proportional to the tube diameter, this leads to the conclusion that the electronic states of larger-diameter nanotubes are more sensitive to hydrostatic pressure. However, besides this general trend, Figure 3 also 
shows a pattern of larger $\left|d E_{11} / d P\right|$ for nanotubes with larger $n-m$ values. A very similar pattern of family grouping has been observed in the transition energies plot [12]. This pattern signifies a chiral angle dependence in addition to the diameter dependence. We found that the pressure coefficient for all the $\mathrm{E}_{11}$ data can be well described by the following equation,

$$
\frac{d E_{11}}{d P}=a \cdot\left(d-d_{0}\right)+b \cdot \cos 3 \alpha,
$$

where $a=-8.3 \mathrm{meV} /(\mathrm{kbar} \cdot \mathrm{nm}), d_{0}=0.68 \mathrm{~nm}$ [17], and $b=-2.5 \mathrm{meV} / \mathrm{kbar}$. In the inset of Fig. 3, the straight line represents the dependence given by Eq.(3). The first term, which is linear in diameter, is related to the fact that the radial compression induced by external pressure is one phase of the nanotube's radial breathing vibrational phonon mode. This mode is experimentally visible in Raman spectra because it has an unusually large resonant intensity enhancement as a result of its strong coupling to electronic excitations from valence to conduction bands [18]. Further studies in this regard may provide more quantitative insights into such electron-phonon coupling as a function of $(n, m)$.

It can be shown that the $d$-dependence of $\mathrm{E}_{11}$ in Eq.(1) scales with the $\mathrm{C}$-C bond length $\left(a_{\mathrm{CC}}\right)$ through the dependence on $a_{\mathrm{CC}}$ of the coefficients in each term [19]. Consequently, if $a_{\mathrm{CC}}$ and $d$ are assumed to deform simply with the same compressive strain under hydrostatic pressure, the bandgap given by Eq.(1) would increase due to the increasing interaction energy $\left(V_{\mathrm{pp} \pi}\right)$ between nearest-neighboring $\mathrm{C}$ atoms [1]. However, the bonds that are circumferential are compressed much more than the ones that lie approximately along the tube axis due to the anisotropy in elastic properties [15]. This perturbs the band structure in a more complex way. Tight binding calculation based on the graphene $\pi$-electron model has shown that the bandgap should increase or decrease depending on the relative shift of the Fermi wave-vector with respect to the nearest $\mathbf{k}$ line in the tube circumferential direction [3]. Therefore, the overall negative pressure coefficient of the bandgap that we observed is likely a higher-order effect beyond the graphene $\pi$-electron model. One possible mechanism that could account for the negative pressure coefficients is the $\sigma^{*}-\pi^{*}$ re-hybridization effect occurring near the large curvature points of nanotubes [20]. It has been demonstrated that the re-hybridization between singlet $\sigma^{*}$ and $\pi^{*}$ conduction bands results in a drastic downward shift of the 
lowest conduction band in small-diameter nanotubes [20] or radially deformed nanotubes [21]. The magnitude of the bandgap reduction depends on the percentage of $\sigma^{*}$-state wavefunction admixed into the lowest conduction band [22]. The re-hybridization effect is the strongest in zigzag nanotubes $(\alpha=0)$ where two of every six $\mathrm{C}$-C bonds are exactly parallel to the tube axis, and vanishes in armchair nanotubes where $\alpha=30^{\circ}[21,23]$. The second lowest conduction band, which is responsible for the $E_{22}$ transitions, is less sensitive to the $\sigma^{*}-\pi^{*}$ re-hybridization [21]. These are consistent with the $\alpha$-dependence of $\left|d E_{11} / d P\right|$ in Eq.(3) and the insensitivity of the $\mathrm{E}_{22}$ peaks to hydrostatic pressure as is shown in Fig. 3. A quantitative explanation of the observed negative and patterned hydrostatic pressure coefficients of SWNT bandgaps requires further theoretical exploration.

In conclusion, by monitoring the shift of the optical absorption peak under hydrostatic pressure, the bandgaps of individual single-walled carbon nanotubes are found to have negative hydrostatic pressure coefficients. The $\mathrm{E}_{22}$ transitions are less sensitive to pressure. Using the assignment of each absorption peak to a specific nanotube $(n, m)$ species, we have determined the pressure coefficient and hydrostatic deformation potential as a function of nanotube structure. The results show a general trend of higher sensitivity of bandgap to hydrostatic pressure for larger-diameter nanotubes, and an additional term that depends on the chirality of the nanotubes.

We thank Prof. S. G. Louie and Prof. R. B. Capaz for helpful discussions. This work was supported by the Director, Office of Science, Office of Basic Energy Sciences, Division of Materials Sciences and Engineering, of the U. S. Department of Energy under Contract No. DE-AC03-76SF00098. J. W. acknowledges support from US NSF Grant No. DMR-0109844. R.B.W. and S.M.B. gratefully acknowledge support from the National Science Foundation (grant CHE-0314270) and the Welch Foundation (grant C-0807). 
References

[1] R. Saito, G. Dresselhaus and M. S. Dresselhaus, Physical Properties of Carbon Nanotubes (Imperial College Press, Singapore, 1998).

[2] J. P. Salvetat, J. M. Bonard, N. H. Thomson, A. J. Kulik, L. Forro, W. Benoit and L. Zuppiroli, Appl. Phys. A, 69, 255 (1999).

[3] Liu Yang and Jie Han, Phys. Rev. Lett, 85, 154 (2000).

[4] T. W. Tombler, C. W. Zhou, L. Alexseyev, J. Kong, H. Dai, L. Liu, C. S. Jayanthi, M. Tang and S. Wu, Nature, 405, 769 (2000).

[5] E. D. Minot, Yuval Yaish,Vera Sazonova, Ji-Yong Park, Markus Brink, and Paul L. McEuen, Phys. Rev. Lett., 90, 156401 (2003).

[6] J. Cao, Q. Wang and H. Dai, Phys. Rev. Lett., 90, 157601 (2003).

[7] S. Kazaoui, N. Minami, H. Yamawaki, K. Aoki, H. Kataura and Y. Achiba, Phys. Rev. B, 62, 1643 (2000).

[8] J. Tang, L. Qin, T. Sasaki, M. Yudasaka, A. Matsushita and S. Iijima, Phys. Rev. Lett. 85, 1887 (2000).

[9] M. J. O’Connell, S. M. Bachilo, C. B. Huffman, V. C. Moore, M. S. Strano, E. H. Haroz, K. L. Rialon, P. J. Boul, W. H. Noon, C. Kittrell, J. Ma, R. H. Hauge, R. B. Weisman, R. E. Smalley, Science, 297, 593 (2002).

[10] Absorption Peak-13 (1.428 eV; width 40meV) is on the border between group B and group A. Since a weak PL peak is also observed near this energy (see inset of Fig. 1), we tentatively place Peak-13 in group A.

[11] The strong emission for group A and the absence of emission for group B are also observed when the sample is excited with a higher photon energy source, the $325 \mathrm{~nm}$ line of a HeCd laser.

[12] S. M. Bachilo, M. S. Strano, C. Kittrell, R. H. Hauge, R. E. Smalley and R. B. Weisman, Science, 298, 2361 (2002).

[13] A. Hagen and T. Hertel, Nano Letters, 3, 383 (2003).

[14] W. F. Kuhs, J. L. Finney, C. Vettier and D. V. Bliss, J. Chem. Phys. 81, 3612(1984).

[15] S. Reich, C. Thomsen and P. Ordejon, Phys. Rev. B, 65, 153407 (2002). 
[16] S.-H. Wei and A. Zunger, Phys. Rev. B 60, 5404 (1999).

[17] We note that the value of $d_{0}$ is close to two times the wall thickness. The origin of $d_{0}$ can be understood from two contributions: 1), a lower limit of the tube diameter where the nanotube is so rigid as to be insensitive to pressure; and 2), A constant, positive contribution to $d E_{11} / d P$ from the increasing C-C interaction energy $\left(V_{\mathrm{pp} \pi}\right)$ due to the C-C bond shrinking under pressure.

[18] A. M. Rao, E. Richter, Shunji Bandow, Bruce Chase, et. al. Science, 275, 187(1997); M. A. Pimenta, A. Marucci, S. D. M. Brown, M. J. Matthews, et. al., J. Mater. Res. 13, 2396 (1998).

[19] R. Saito, G. Dresselhaus and M. S. Dresselhaus, Phys. Rev. B, 61, 2981 (2000).

[20] X. Blase, L. X. Benedict, E. L. Shirley and S. G. Louie, Phys. Rev. Lett. 72, 1878 (1994).

[21] J. C. Charlier, Ph. Lambin and T. W. Ebbesen, Phys. Rev. B, 54, 8377 (1996).

[22] H. Yorikawa and S. Muramatsu, Phys. Rev. B, 52, 2723 (1995).

[23] A. Kleiner and S. Eggert, Phys. Rev. B 64, 113402 (2001). 


\section{Figure Captions}

Fig. 1 Absorption spectrum of a SWNT suspension. Inset shows the absorption spectrum in the near infrared region in comparison with a PL spectrum. No PL peak was observed above $1.5 \mathrm{eV}$ (not shown here). The peaks are numbered in the order of increasing peak energy.

Fig. 2 (a) Absorption spectrum in the near infrared (group A) taken at a range of hydrostatic pressures. The curves are vertically offset for clarity. (b) Absorption curves at higher energies (group B) as a function of pressure.

Fig. 3 Measured linear pressure coefficients as a function of peak energy. The number beside each data point shows the peak number. Numbers in the $E_{11}$ symbols are the value of $n-m$. The dashed lines connect families with equal $n-m$ values. Inset shows the dependence given by Eq.(3) for all $\mathrm{E}_{11}$ points. 
Table and table caption

\begin{tabular}{cccccccc}
\hline $\begin{array}{c}\mathbf{E}_{\mathbf{1 1}} \\
\text { peak }\end{array}$ & $\begin{array}{c}\mathbf{E}_{\mathbf{1 1}} \\
(\mathbf{e V})\end{array}$ & $\begin{array}{c}\mathbf{d E}_{\mathbf{1 1}} / \mathbf{d P} \\
(\mathbf{m e V} / \mathbf{k b a r})\end{array}$ & $\begin{array}{c}\mathbf{d E}_{\mathbf{1 1}} / \mathbf{d} \mathbf{d n V} \\
(\mathbf{e V})\end{array}$ & $\begin{array}{c}\text { Assignment } \\
(\mathbf{n}, \mathbf{m})\end{array}$ & $\begin{array}{c}\mathbf{E}_{\mathbf{2 2}} \\
\mathbf{p e a k}\end{array}$ & $\begin{array}{c}\mathbf{E}_{\mathbf{2 2}} \\
(\mathbf{e V})\end{array}$ & $\begin{array}{c}\mathbf{d E}_{\mathbf{2 2}} / \mathbf{d P} \\
(\mathbf{m e V} / \mathbf{k b a r})\end{array}$ \\
\hline 1 & 0.899 & & & $(9,8)$ & 14 & 1.543 & -0.3 \\
\hline 2 & 0.933 & & & $(12,2)$ & 16 & 1.828 & \\
\hline 3 & 0.977 & -2.9 & 6.4 & $(8,7)$ & 15 & 1.700 & -1.3 \\
\hline 4 & 0.994 & & & $(11,1)$ & 19 & 2.083 & -0.7 \\
\hline 5 & 1.035 & -4.6 & 10.3 & $(11,3)$ & 14 & & \\
\hline 6 & 1.054 & -3.0 & 7.0 & $(8,6)$ & 15 & & \\
\hline 7 & 1.105 & -2.0 & 4.9 & $(7,6)$ & 17 & 1.917 & -1.6 \\
\hline 8 & 1.123 & & & $(9,4)$ & 15 & & \\
\hline 9 & 1.173 & -3.8 & 9.5 & $(10,2)$ & 15 & & \\
\hline 10 & 1.209 & -1.8 & 4.7 & $(7,5)$ & 17 & & \\
\hline 11 & 1.272 & -1.0 & 2.8 & $(6,5)$ & 20 & 2.239 & -0.4 \\
\hline 12 & 1.295 & & & $(8,3)$ & 17 & & \\
\hline 13 & 1.428 & -0.6 & 2.1 & $(6,2)$ & 24 & 3.030 & \\
\hline & & & & 18 & 2.043 & \\
\hline & & & & 21 & 2.456 & 0.1 \\
\hline & & & & 22 & 2.594 & -1.5 \\
\hline
\end{tabular}

Tab. I Absorption peak energies, linear pressure coefficients, deformation potentials and corresponding nanotube assignments. 


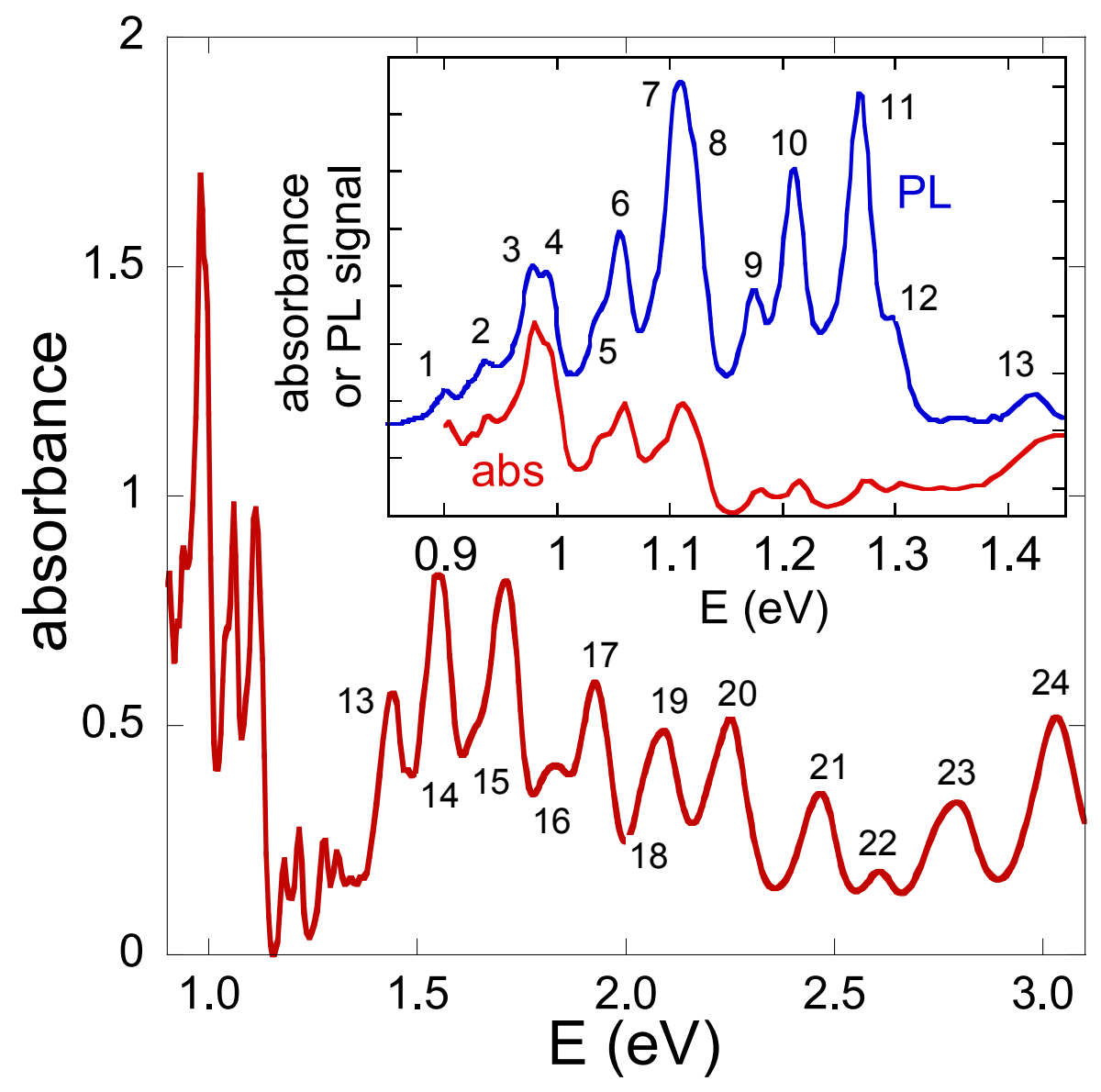

Fig. 1 of 3

J. Wu et. al. 


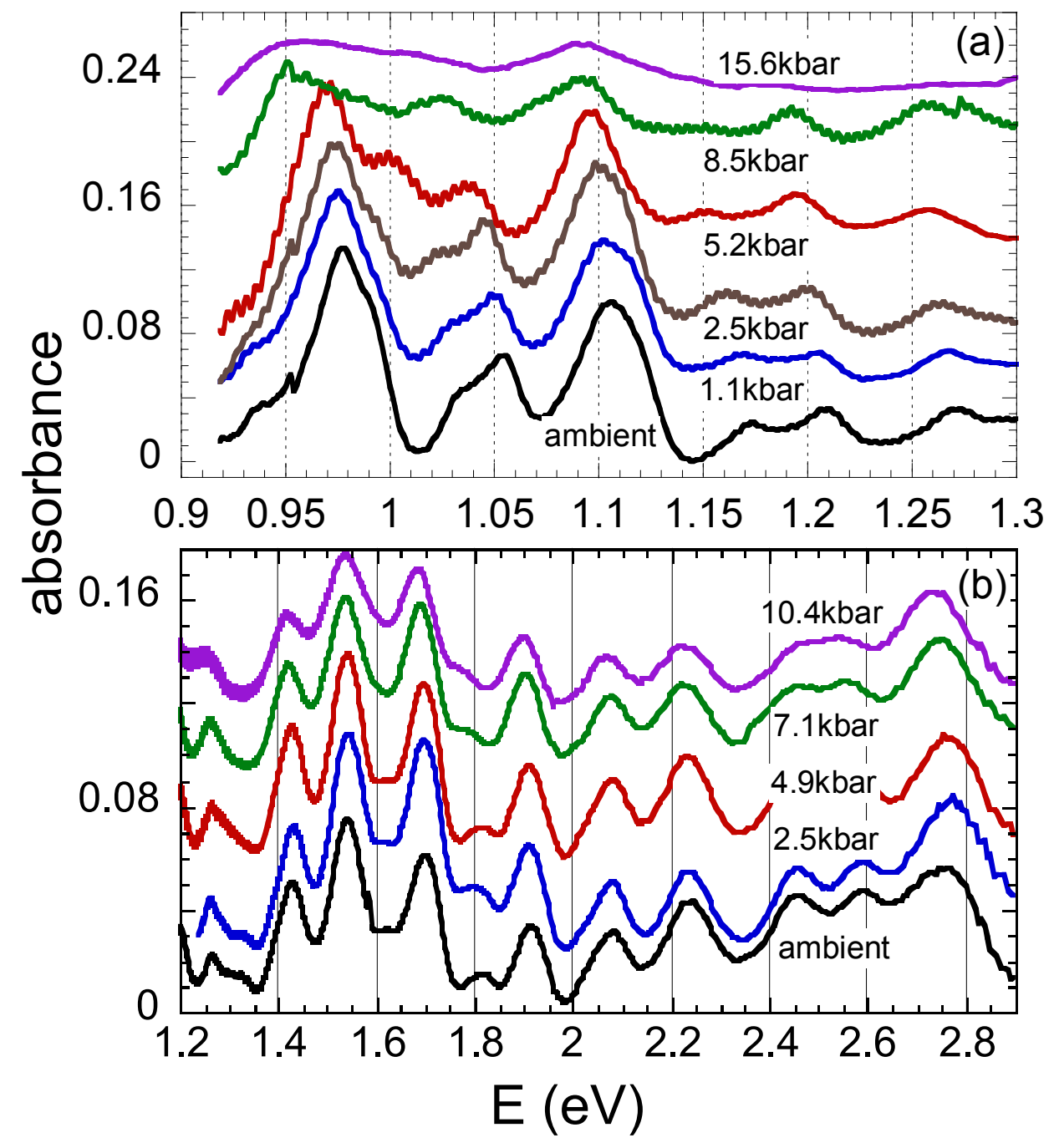

Fig. 2 of 3

J. Wu et. al. 


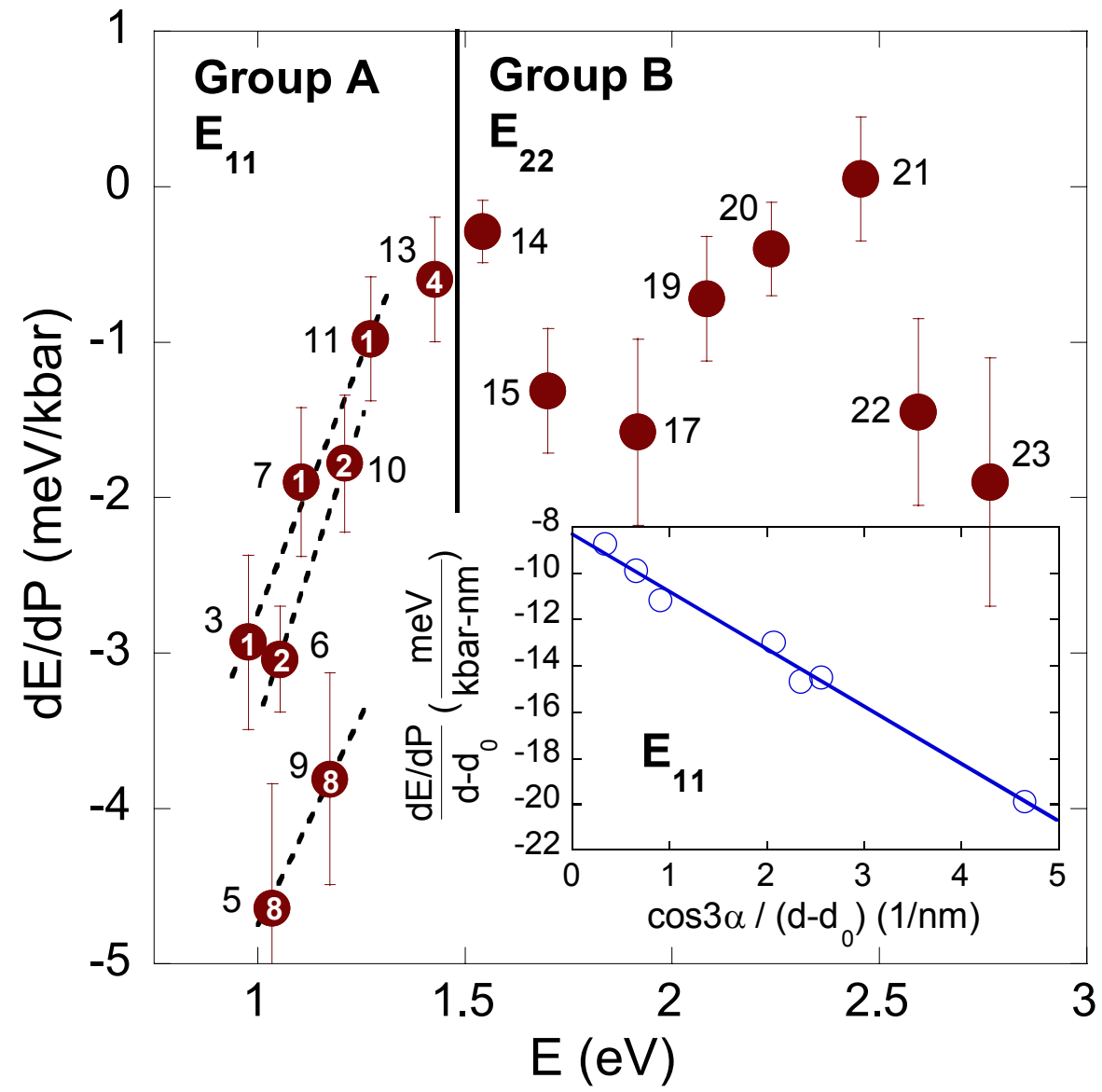

Fig. 3 of 3

J. Wu et. al. 